\title{
Sixty-year perspective on coronary artery bypass grafting in women
}

\author{
Renee S. Hartz, MD \\ Julie A. Swain, MD \\ Lynda Mickleborough, $\mathrm{MD}^{\mathrm{b}}$
}

See related editorials on pages 617 and 618.
From the Department of Surgery, Tulane University Health Sciences Center, New Orleans, La, ${ }^{a}$ and The Toronto Hospital, Toronto, Ontario, Canada. ${ }^{\mathrm{b}}$

Received for publication June 18, 2003; accepted for publication July 7, 2003.

Address for reprints: Renee S. Hartz, MD, Department of Surgery, Tulane University Health Sciences Center, 1430 Tulane Ave, New Orleans, LA 70112-2699 (E-mail: rhartz@tulane.edu).

J Thorac Cardiovasc Surg 2003;126:620-2

Copyright $(9) 2003$ by The American Association for Thoracic Surgery

$0022-5223 / 2003 \$ 30.00+0$

doi:10.1016/S0022-5223(03)01201-7

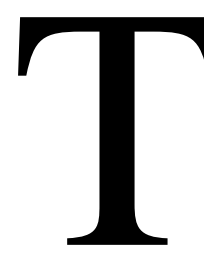

he " 60 " in our title was designed to get the reader's attention, since the first coronary artery bypass (CABG) procedure was performed less than 40 years ago. This editorial was written by three women cardiac surgeons, each of whom has practiced for 20 years, and it represents our 60-year cumulative experience. We are pleased to make a contribution to this timely editorial series and have highlighted key events in the history of recognition and treatment of coronary artery disease in women.

\section{The Eighties}

Despite the fact that the systematic study of risk factors for coronary artery disease (CAD) had begun 30 years earlier (spearheaded by the Framingham Heart Study), CAD was still considered a disease of men. Early in this decade, the suspicion that women were referred for interventional therapies too seldom and too late led to the initiation of several retrospective reviews of referral and treatment patterns in women and men. At the same time the treatments were improving dramatically; there was an exponential increase in the performance of CABG, use of the internal thoracic artery as a bypass graft was becoming routine in many programs, and coronary angioplasty was rapidly evolving. The perception that women did worse after CABG was common, but few studies documented this fact.

In the Framingham Study, the diagnosis of CAD was based on the presence of chest pain. After receiving this diagnosis, significantly fewer women than men went on to have adverse cardiac events. However, the Coronary Artery Surgery Study (CASS), in which all patients underwent coronary angiography, showed that the prevalence of CAD in women with definite angina was $72 \%$. ${ }^{1}$ Subsequent reanalysis of the Framingham data after the CASS findings were published revealed that older women who had angina in Framingham (aged 60-69 years) had the same incidence of adverse events as did men. This was the first large study documenting the influence of age on CAD in women and specifically dispelling the notion that CAD rarely develops in women. ${ }^{2}$

In the late 1980s a major effort was undertaken by the American Heart Association (AHA) to increase awareness of heart disease in women. Numerous articles documenting both under-diagnosis and under-referral in women, even after a positive stress test had been obtained, were now available and became widely quoted. Furthermore, it appeared that the results of all interventional treatments were significantly worse in women. The AHA then launched a national campaign to address these issues and to improve the recognition and treatment of CAD in women. In 1989 the AHA sponsored the first national conference on heart disease in women. In her introductory comments, the Honorable Constance Horner, under secretary of the US Department of Health and Human Services, stated, "the time has come to initiate a nationwide effort to bring the risk of heart disease to the attention of women."

Some investigators questioned whether the perceived "referral bias" actually represented more appropriate treatment in women than in men. ${ }^{3}$ For example, 
low-risk women were less likely to be referred for CABG than low-risk men. With high-risk patients, however, men and women were equally likely to be referred. Stated another way, among patients for whom treatment would offer little survival benefit, men were more likely to be referred than women, with the reverse trend being observed in women. In the ensuing few years, dozens of state and local chapters of the AHA held educational conferences on heart disease in women to begin to address these complex questions. A National Institutes of Health initiative was also launched, and inclusion of appropriate representation of women patients became a condition of funding research grants.

In 1986 HCFA (the Health Care Financing Agency) released non-risk-adjusted "data" from cardiac surgery programs and individual surgeons. The cardiac surgical community, accustomed to intense scrutiny because of the high profile nature of the specialty and the huge expenditure of health care dollars on cardiac procedures, was stunned by HCFA's action. A strong disincentive was created to operate on "sick" patients, which may have adversely affected the availability of CABG to women. Unfortunately, many practices began either to refuse high-risk patients or to send them to tertiary referral centers. Other programs with good results began to use their data for advertising purposes, a new phenomenon in cardiac surgery. ${ }^{4}$ Fortunately, the HCFA report also encouraged the development of several local and national methods of risk stratification. Cardiac surgeons became pioneers in risk stratification in order to create "a level playing field" and to promote members of the specialty who were willing to care for very sick patients.

\section{The Nineties}

In 1990, just 1 year after the AHA conference on heart disease in women, national mortality rates for cardiovascular diseases (heart attack and stroke) were greater for women than men for the first time. ${ }^{5}$ In this decade, preoperative risk stratification and postoperative outcomes analysis were widely adopted by cardiac surgery programs. Moreover, a description of the risk-benefit ratio became an integral component of informed consent. The factors responsible for the increased mortality rates in women could now be determined and prospective studies were initiated.

Several different risk-stratification systems were developed. Older age, hypertension, diabetes, renal failure, nonelective operation, and depressed left ventricular function were all shown to be significant factors in predicting adverse outcome (mortality and morbidity) after CABG. Each of these attributes, except left ventricular dysfunction, was shown to be significantly more common in women than in men. Although the comparative weight of each risk factor varied in different models, women were shown to have approximately twice the operative mortality of men in al- most every published series. Only when high-risk patients of both genders were matched for age, comorbidity, and acuity did this difference in operative mortality disappear. Thus, in this decade the long-held perception that women undergoing $\mathrm{CABG}$ are generally sicker than their male counterparts, and that they have worse outcomes, was statistically verified.

Although older age has always been recognized as a predictor of worse outcomes, the answer to the question "how old is old" is a moving target, especially with reference to CAD. The AHA has confirmed that CAD-related mortality increases 3-fold to 5-fold with each decade of age. ${ }^{5}$ In the general population the mean life expectancy of women increased to almost 80 years, and in CABG series women are significantly older than men because signs and symptoms of CAD appear at least 10 years later. Older age has emerged as an independent predictor of operative mortality for cardiac surgical procedures in women. Although the average age of patients in the Society of Thoracic Surgeons database has remained the same since 1990 when it was 64 years, women undergoing CABG have always been older. In 1995, men were 63.6 years old and women were 66.9. Currently, men are 64.3 and women are 67.4 years old. ${ }^{6}$ Cardiovascular diseases have become the leading cause of death in American women, killing more women than cancer, accidents, and diabetes combined. ${ }^{5}$ One third of women older than 65 have clinical manifestations of CAD. Although the overall percentage of women in CABG series has remained relatively stable at around $30 \%$, octogenarian series often include $40 \%$ to $50 \%$ women. These elderly patients frequently have serious comorbid conditions. Outcome studies are needed to determine which combinations of age-related and comorbid conditions lead to an unacceptable risk-benefit ratio for $\mathrm{CABG}$.

\section{The Twenty-First Century}

We are entering an era of geriatric medical care. The oldest of the baby-boom generation will turn 65 years old in 2011, and 1 out of 5 Americans will be 65 or older by 2030 . Twenty years later, in 2050, 19 million Americans will be older than $85 .^{7}$ Unfortunately, geriatric specialists are in critically short supply. Therefore, the American Geriatric Society has challenged physicians of many specialties, including thoracic surgery, to increase their knowledge of geriatric medicine and to include geriatrics in medical school and residency curricula.

We must face the certainty that, because of the aging population, morbidity and mortality rates after CABG will soon begin to increase. We must accept the tremendous challenges associated with operating on older and sicker patients and alter our perception of the typical CABG candidate: she is, in fact, a 78-year-old woman. 


\section{References}

1. Kennedy JW, Killip T, Fisher LD, et al. The clinical spectrum of coronary artery disease and its surgical and medical management, 1974-1979: The Coronary Artery Surgery Study. Circulation. 1982; 66(Suppl III):III-16.

2. Lerner DJ, Kannel WB. Patterns of coronary heart disease morbidity and morality in the sexes: a 26-year follow-up of the Framingham population. Am Heart J. 1986;11:383-90.

3. Bickell NA, Pieper KS, Lee KL, et al. Referral patterns for coronary artery disease: bias or good clinical judgment? Ann Intern Med. 1992; 1166:791-7.

4. Burack JH, Impellizzeri P, Homel P, Cunningham JN Jr. Public reporting of surgical mortality: a survey of New York State cardiothoracic surgeons. Ann Thorac Surg. 1999;68:1195-202.

5. American Heart Association. Heart and stroke facts: 1996 statistical supplement. Dallas: American Heart Association; 1996.

6. The Society of Thoracic Surgeons National Cardiac Database.

7. The American Geriatric Society Database. 